\title{
Enhancing IEEE 802.11n WLANs using group-orthogonal code-division multiplex
}

\author{
Guillem Femenias and Felip Riera-Palou \\ Mobile Communications Group - Dept. of Mathematics and Informatics \\ University of the Balearic Islands \\ 07122 Palma, Mallorca (Illes Balears), Spain. \\ \{guillem.femenias, felip.riera\}@uib.es
}

\begin{abstract}
The definition of the next generation of wireless networks is well under way within the IEEE 802.11 High Throughput Task Group committee. The resulting standard, to be called IEEE $802.11 \mathrm{n}$, is expected to be a backward-compatible evolution of the successful IEEE $802.11 \mathrm{a} / \mathrm{g}$ systems also based on multicarrier techniques. It can be anticipated that $802.11 \mathrm{n}$ systems will outperform its predecessors in terms of transmission rate and/or performance, mainly, due to the use of multiple antennae technology for transmission and reception. In this paper we propose to incorporate group-orthogonal (GO) code division multiplex (CDM) into the IEEE 802.11n specifications to further enhance its performance. It is shown how GO-CDM can take full advantage of the diversity offered by the multiple antennae and multicarrier transmission by using an iterative maximum likelihood (ML) joint detector. Furthermore, the use of GO-CDM does not compromise the backward compatibility with legacy systems.
\end{abstract}

Keywords: IEEE $802.11 \mathrm{n}$, multicarrier, code-division multiplex, maximum likelihood detection, turbo receivers.

\section{Introduction}

The last decade has seen an explosive growth in the deployment of wireless local area networks (WLANs) which has made the concept of nomadic computing a reality. Most of these networks are based on one of the flavours of the IEEE 802.11 family of standards. The most recent and powerful set of specifications currently deployed, IEEE $802.11 \mathrm{~g}$, operates on a bandwidth of $20 \mathrm{MHz}$ and its physical layer is based on a particular form of multicarrier transmission, namely, orthogonal frequency division multiplexing (OFDM) enabling these systems to achieve transmission rates up to $54 \mathrm{Mbps}$. At present, the standardisation of what should be the next step, named IEEE $802.11 \mathrm{n}$, is being pursued by the IEEE 802.11 High Throughput Task Group committee. The new standard will support much higher transmission rates thanks to the use of multiple antenna technology and other enhancements such as the possibility of operating on a

Please use the following format when citing this chapter:

Femenias, G., Riera-Palou, F., 2007, in IFIP International Federation for Information Processing, Volume 245, Personal Wireless Communications, eds. Simak, B., Bestak, R., Kozowska, E., (Boston: Springer), pp. 184-195. 
$40 \mathrm{MHz}$ bandwidth (employing more subcarriers) and transmission modes using a reduced guard interval. In its fastest mode, $802.11 \mathrm{n}$ is expected to surpass a transmission rate of $700 \mathrm{Mbps}$. Despite all the enhancements introduced, it is mandatory for the new standard to remain compatible with multicarrier legacy systems $(802.11 \mathrm{a} / \mathrm{g})$ and therefore, $802.11 \mathrm{n}$-compliant devices should have means to fall back to older 802.11 specifications.

Like its predecessors, $802.11 \mathrm{a}$ and $802.11 \mathrm{~g}$, the new $802.11 \mathrm{n}$ standard will also be based on OFDM allowing the use of other enhancements which have recently been proposed for this type of physical layers. A powerful improvement over conventional OFDM was the introduction of multicarrier code division multiplex (MC-CDM) OFDM by Kaiser in [1]. In MC-CDM, rather than transmitting a single symbol on each subcarrier as in conventional OFDM, groups of symbols are multiplexed together by means of orthogonal spreading codes and simultaneously transmitted on a group of subcarriers. This technique resembles very much the principle behind multicarrier code-division multiple access (MC-CDMA) [2] where different users share a group of subcarriers by using each of them a different spreading code. More recently, group-orthogonal MCCDMA (GO-MC-CDMA) $[3,4]$ has been introduced as a particular flavour of MC-CDMA whereby users are split in groups and each group exclusively uses a (small) subset of all the available subcarriers. The subcarriers forming a group are chosen to be as separate as possible in the available bandwidth in order to maximise the frequency diversity gain. A GO-MC-CDMA setup can be seen as many independent MC-CDMA systems of lower dimension operating in parallel. This reduced dimension allows the use of optimum receivers for each group based on maximum likelihood detection at a reasonable computational cost. Grouporthogonality has also been proposed for (uncoded) MC-CDM systems in [5] where results are given for group dimensioning and spreading code selection. Nevertheless, as shown in [6], the benefits of CDM-OFDM are rather limited when measuring the coded performance in typical operating scenarios conforming to IEEE 802.11a specifications, especially, when using decoders based on soft/iterative procedures. This is due to the large subcarrier correlation found in many wireless environments which severely limits the achievable frequency diversity. This issue is largely solved with the introduction of multiple transmit and receive antennae as then subcarrier correlation within a group can be greatly reduced by exploiting the spatial dimension. A comparison between two of the possible techniques to exploit the spatial diversity, namely, space-time block coding (STBC) and cyclic delay diversity (CDD), within the context of IEEE 802.11n has recently appeared in [7] but in there, the receiver structures proposed are based on linear techniques (minimum mean square error detectors) and hence are not suitable for iterative processing.

This paper, after reviewing the physical layer of the current IEEE $802.11 \mathrm{n}$ draft, proposes the use of GO-MC-CDM to further improve its performance. It will be shown how using an iterative reception scheme based on a maximum likelihood multisymbol detector (ML-MSD) with soft decoding allows the exploitation of both, frequency and spatial diversity. Simulation results are presented for 
two possible spatial configurations, one uniquely based on cyclic delay diversity (CDD) and another one based on the combination of spatial division multiplexing (SDM) and CDD.

We conclude this introduction with a brief notational remark: throughout this paper vectors and matrices are denoted with lower and upper cases bold characters, respectively. Scalars are represented with non-bold characters (either lower or upper case). We use the notation $\mathcal{D}(\mathbf{x})$ to denote a diagonal matrix with vector $\mathbf{x}$ at its main diagonal. Vectors are assumed to be column-oriented and the notation $(\cdot)^{T}$ is used to denote the transposition of a vector or matrix. Finally, $\mathbf{x}_{[k]}$ represents a vector $\mathbf{x}$ with its $k$ th entry removed.

\section{System model for current IEEE 802.11 n proposal}

The current draft for the physical layer of IEEE 802.11n can be found in [8]. As mentioned in the introduction, a qualitative difference of IEEE 802.11n with respect to previous standards is the introduction of multiple antenna technology. Moreover, the standard is defined in such a way that allows the use of different methods to exploit the spatial dimension, namely, spatial division multiplexing (SDM), cyclic delay diversity (CDD) and space-time block coding (STBC). In this work we have focused on the design of an enhanced physical layer based on the application of GO-CDM when using CDD and/or SDM ${ }^{1}$. The transmission architecture is depicted in Fig. 1. Note that this figure already includes the optional GO-CDM extension (in dashed lines) which will be covered in detail in the next section.

We focus on the IEEE 802.11 n specification operating in a bandwidth of $B=20$ $\mathrm{MHz}$ utilized by means of $N_{c}=64$ orthogonal subcarriers of which $N_{c}^{d}=52$ subcarriers are used to transmit user data while the rest, $N_{c}^{p}=12$, correspond to pilot and guard subcarriers. Transmitter and receiver are assumed to have $N_{t}$ and $N_{r}$ antennas, respectively, with $1 \leq N_{t}, N_{r} \leq 4$.

The user data to be transmitted is generated and segmented into frames of $N_{b}$ bits. It is assumed that frames are independent of one another, and therefore, to simplify notation, there is no need to specify the segment index. Each generated frame is subsequently fed to a rate $R_{c}$ punctured convolutional encoder (RCPCC box in Fig. 1) to generate $N_{b} / R_{c}$ coded bits. The coded bits are then separated into $N_{s} \in\{1,2,3,4\}$ spatial streams as specified by the spatial parsing equation in [8]. It should be pointed out that for the cases of employing CDD and/or SDM, it should hold that $N_{s} \leq N_{t}$. The bits on each stream are then interleaved (blocks $\Pi_{1}$ in Fig. 1) and subsequently mapped to symbols from an $m$-point constellation (BPSK, m-QAM) with $m=2^{M}$ where $M$ represents the number of bits per symbol, yielding the set of signal vectors to be transmitted $\mathbf{s}^{w}=$ $\left(\begin{array}{llll}s_{1}^{w} & s_{2}^{w} & \ldots & s_{N_{\mathrm{qam}}}^{w}\end{array}\right)^{T}$ where $1 \leq w \leq N_{s}$ represents the spatial stream index and $N_{\text {qam }}=N_{b} /\left(R_{c} N_{s} M\right)$ denotes the number of symbols per frame to be

${ }^{1}$ The application of GO-CDM in combination with STBC in the context of IEEE $802.11 \mathrm{n}$ is a topic of current research. 
transmitted on each spatial stream. The symbols on the different spatial streams are at this point properly segmented into $N_{\text {OFDM }}$ OFDM symbols, each made of $N_{d}$ (QAM) symbols holding that $N_{\text {qam }}=N_{c}^{d} N_{O F D M}$. We will denote by $\mathbf{s}_{l}^{w}$, $1 \leq l \leq N_{O F D M}$, the $l$ th OFDM symbol of the $w$ th spatial stream in the current frame. Skipping for the moment, the GO-CDM processing, the resulting OFDM symbols from all streams are linearly mixed according to

$$
\left(\mathbf{x}_{l}^{u}\right)^{T}=\boldsymbol{W}\left(\mathbf{s}_{l}^{1} \mathbf{s}_{l}^{2} \ldots \mathbf{s}_{l}^{N_{s}}\right)^{T}
$$

where $1 \leq u \leq N_{t}$ and $W$ represents an $N_{t} \times N_{s}$ spatial spreading matrix. The role of the matrix $W$ is twofold: on one hand, for the cases where $N_{s}<N_{t}$, the spatial spreading serves to distribute the incoming streams among all transmit antennae thus making full use of the available spatial diversity. On the other hand, the specific selection of $\boldsymbol{W}$, in combination with a suitable choice of the cyclic delays $\delta_{1}, \ldots, \delta_{N_{t}}$ (see Fig. 1), provides the system with either CDD, SDM or a combination of the two. Examples are later provided showing how this matrix and cyclic delays can be chosen to exploit the spatial diversity in a prescribed manner. Finishing the transmission procedure, and as shown in Fig. 1, the different spatially spread OFDM symbols, $\mathrm{x}_{l}^{u}$, are then IFFT-converted, expanded with a cyclic prefix and processed by the RF transmission chains.

Taking for granted that transmit and receive antennas are sufficiently apart, the $N_{t} \times N_{r}$ propagation channels between transmitter and receiver are safely assumed to be independent and derived from a common and scenario-dependent power delay profile

$$
S(\tau)=\sum_{p=0}^{P-1} \phi(p) \delta\left(\tau-\tau_{p}\right)
$$

where $P$ denotes the number of independent paths of the profile and $\phi(p)$ and $\tau_{p}$ denote the power and delay of each path, respectively. A single realization of the channel impulse response between transmit antenna $a_{t}$ and receive antenna $a_{r}$ at time instant $t$ will then have the form

$$
h^{a_{t}, a_{r}}(t ; \tau)=\sum_{p=0}^{P-1} h_{p}^{a_{t}, a_{r}}(t) \delta\left(\tau-\tau_{p}\right)
$$

where it will hold that $E\left[\left|h_{p}^{a_{t}, a_{r}}(t)\right|^{2}\right]=\phi(p)$. The corresponding frequency response will be given by

$$
\bar{h}^{a_{t}, a_{r}}(t ; f)=\sum_{p=0}^{P-1} h_{p}^{a_{t}, a_{r}}(t) \exp \left(-j 2 \pi f \tau_{p}\right)
$$

which, evaluated at the $N_{c}$ equispaced subcarrier frequencies, $f_{0}, f_{1}, \ldots, f_{N c-1}$, yields the $N_{c} \times 1$ vector $\overline{\mathbf{h}}^{a_{t}, a_{r}}(t)=\left(\bar{h}^{a_{t}, a_{r}}\left(t ; f_{0}\right) \ldots \bar{h}^{a_{t}, a_{r}}\left(t ; f_{N_{c}-1}\right)\right)^{T}$. Assuming without loss of generality that the channel is static over the duration of a frame and independent from other frames, to simplify the notation, we will 


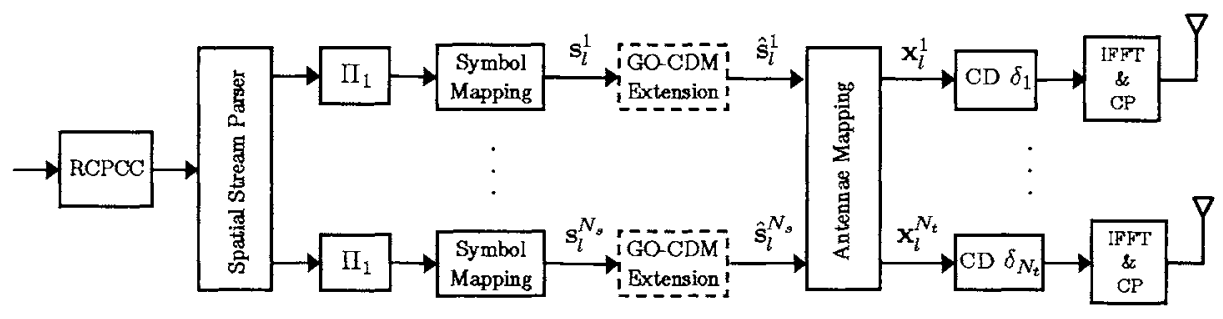

Fig. 1. IEEE 802.11n transmitter proposal supporting SDM and/or CDD.

express the frequency response of the data subcarriers (i.e. pilots and guard subcarriers excluded) simply as $\overline{\mathrm{h}}^{a_{t}, a_{r}}=\left(\bar{h}_{0}^{a_{t}, a_{r}} \bar{h}_{1}^{a_{t}, a_{r}} \ldots \bar{h}_{N_{c}^{d}-1}^{a_{t}, a_{r}}\right)^{T}$.

At this point it is necessary to distinguish which form of spatial diversity is employed, which is basically determined by the choice of $\boldsymbol{W}$ and cyclic delays. In setups based exclusively on SDM, it will hold that $N_{s}=N_{t}$ and $\delta_{a_{t}}=0$ for $1 \leq a_{t} \leq N_{t}$. This implies that each antenna is in charge of transmitting one spatial stream. In contrast, in configurations where transmit antennae are configured to exploit CDD, it will hold that $N_{s}=N_{t} / \Lambda$, where $\Lambda$ denotes the number of antennae involved in the CDD processing (CDD factor) of each spatial stream. That is, when using CDD, the available antennae are split in $N_{s}$ groups of $\Lambda$ antennae and each group is used for the transmission of one spatial stream. In CDD, all the antennae in a CDD group transmit the same information but each antenna applies a different cyclic delay [9]. It is easy to show [9] that this amounts to transmit a given spatial stream information from a single antenna over the composite channel reaching receive antenna $a_{r}$ given by

$$
\overline{\mathbf{h}}_{w}^{a_{r}}=\sum_{a_{t}^{w}=0}^{\Lambda \cdots 1} \overline{\mathbf{h}}^{a_{t}^{w}, a_{r}} \exp \left(-j \frac{2 \pi \delta_{a_{t}^{w}}}{N_{c}}\right) .
$$

where $\overline{\mathbf{h}}^{a_{t}^{w}, a_{r}}$ denotes the different channel frequency responses between the antennae belonging to CDD group $w$ and receiver antenna $a_{r}$ and $\delta_{a_{t}^{w}}$ represents the specific cyclic delay applied by each antenna in the CDD group. Taking into account that the available transmit antennae will be split into parallel CDD systems, it can be derived from [9] that an advantageous delay choice will be given by

$$
\delta_{a_{t}}=\left\{\begin{array}{cl}
0 & , \text { if } \bmod \left(a_{t}-1, \Lambda\right)=0 \\
N_{c} /\left(N_{t} / \Lambda\right)+\delta_{a_{t}-1} & , \text { otherwise }
\end{array}\right.
$$

where $\bmod (a, b)$ denotes the modulus of $a / b$. It is important to stress that, to all effects, the composite CDD channel $\bar{h}_{w}^{a_{r}}$ can be considered as a conventional channel with the distinctive feature of an increased frequency selectivity with respect to the original power profile defined by $S(\tau)$.

Having defined the transmission scheme and channel, it is now possible to specify 


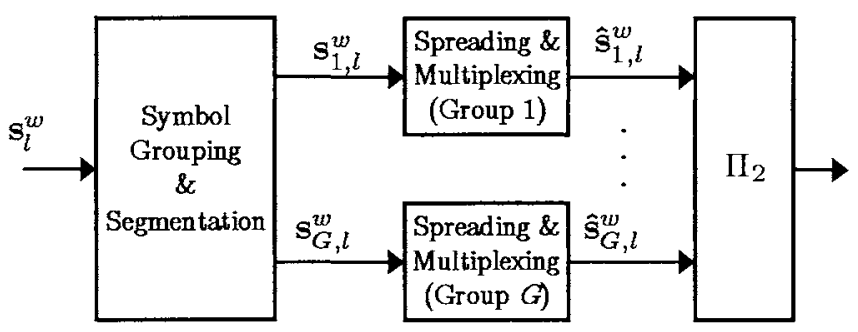

Fig. 2. GO-CDM extension.

the reception equation for the $N_{s}$ OFDM-symbols which have been transmitted in parallel at a given instant as

$$
\left(\begin{array}{c}
\mathbf{r}_{l}^{1} \\
\vdots \\
\mathbf{r}_{l}^{N_{r}}
\end{array}\right)=\left(\begin{array}{ccc}
\mathbf{H}^{1,1} & \ldots & \mathbf{H}^{1, N_{s}} \\
\vdots & \ddots & \vdots \\
\mathbf{H}^{N_{r}, 1} & \ldots & \mathbf{H}^{1, N_{s}}
\end{array}\right)\left(\begin{array}{c}
\mathbf{x}_{l}^{1} \\
\vdots \\
\mathbf{x}_{l}^{N_{s}}
\end{array}\right)+\left(\begin{array}{c}
\boldsymbol{v}_{l}^{1} \\
\vdots \\
\boldsymbol{v}_{l}^{N_{r}}
\end{array}\right)
$$

where $\mathbf{H}^{i, j}$ will be of the form $\mathcal{D}\left(\bar{h}^{a_{r}, a_{t}}\right)$ for the case of $\operatorname{SDM}\left(N_{t}=N_{s}\right)$ or the form $\mathcal{D}\left(\bar{h}_{w}^{a_{r}}\right)$ for the case where CDD is used $\left(N_{s}=N_{t} / \Lambda\right)$. The $N_{c}^{d} \times 1$ vectors $v_{g, l}^{1}, \ldots, v_{g, l}^{N_{r}}$ represent the additive white Gaussian noise samples on each receiving antenna. Every scalar noise sample, is assumed to be distributed according to a zero-mean Gaussian pdf with variance $\sigma_{n}^{2}$. The original data symbols to be transmitted $s_{i}^{w}$, are suitably scaled to have power $E\left\{\left|s_{i}^{w}\right|^{2}\right\}=1$ in the case of SDM transmission and $E\left\{\left|s_{i}^{w}\right|^{2}\right\}=1 / \Lambda$ if the CDD component is present. In this latter case, this factor represents the distribution of energy available per symbol among the various transmit antennas used for the transmission of the $w$ th spatial stream. For both cases, SDM and CDD (and their combinations), this power normalisation allows the operating signal to noise ratio to be written as $E_{s} / N_{0}=1 / \sigma_{n}^{2}$.

\section{Group-orthogonal CDM for IEEE 802.11n}

The introduction of multiple transmit and receive antenna brings along the possibility of further exploiting the potential frequency selectivity, specially, whenever a CDD component is present. To this end, GO-CDM can be used to distribute the source symbols energy among multiple subcarriers. Figure 2 shows a detailed diagram of the GO-CDM mechanism which would correspond to the dashed boxes in Fig. 1. The GO-CDM processing is performed on each spatial stream and consists of splitting the incoming OFDM symbols, $\mathrm{s}_{l}^{w}$, into $G$ groups of $Q=N_{c}^{d} / G$ QAM symbols each. Each group, $\mathbf{s}_{g, l}^{w}$, is then assigned $Q$ subcarriers which will be used to jointly transmit the $Q$ source symbols in the group. The distribution of the group symbols among the $Q$ subcarriers is carried out by means of a 


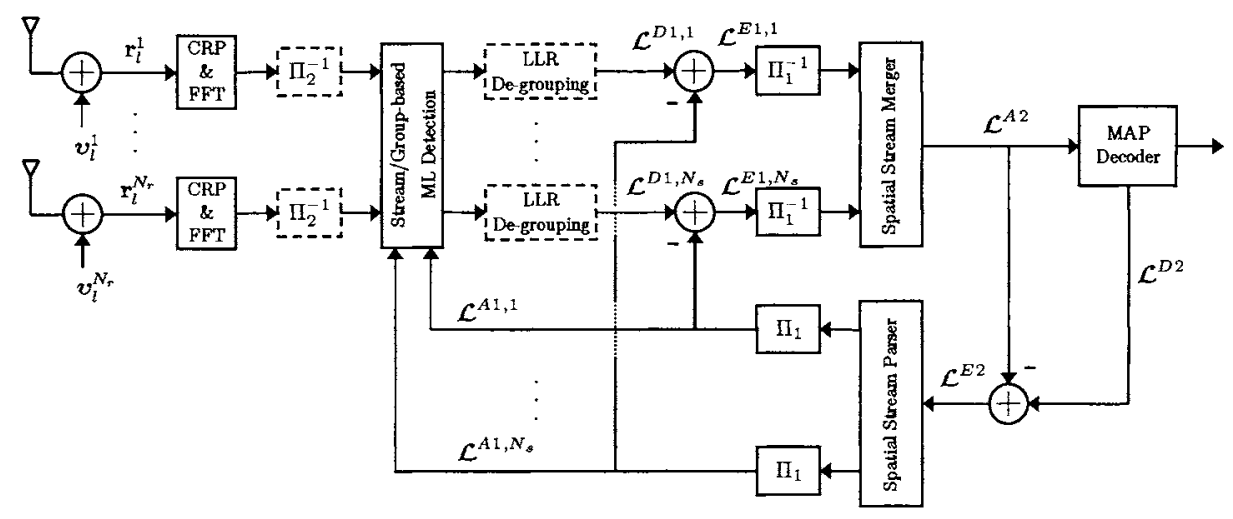

Fig. 3. ML-based turbo receiver for GO-CDM enhanced IEEE 802.11n.

spread and multiplex operation defined by

$$
\hat{\mathbf{s}}_{g, l}^{w}=\mathbf{C ~ \mathbf { s } _ { g , l } ^ { w }}
$$

with $1 \leq g \leq G$ and with $\mathbf{C}=\left(\mathbf{c}^{0} \mathbf{c}^{1} \ldots \mathbf{c}^{Q-1}\right)$ representing the $Q \times Q$ spreading matrix with each $\mathrm{c}^{q}$ denoting the $Q \times 1$ spreading code associated with the $q^{t h}$ symbol in the $g^{t h}$ group. A typical choice of spreading matrix is the WalshHadamard matrix due to its column-wise orthogonality and low complexity implementation. We note that the spreading matrix is common to all groups since each group utilizes separate (and orthogonal) sets of subcarriers and therefore, there is no need to provide extra separation. The resulting spread and multiplexed symbols from all groups are then time interleaved (block $\Pi_{2}$ in Fig. 2) to increase the resilience against noise. The idea is to transmit (QAM spread) symbols from different OFDM-symbols in each group so that, upon deinterleaving at the receiver, each symbol in the group has experienced uncorrelated noise samples. To avoid confusion with the spatial spreading performed by the linear transformation given by $\boldsymbol{W}$, from this point onwards, we will refer to the GO-CDM processing as temporal spreading. As can be inferred from Fig. 1, if GO-CDM processing is present, the spatial spreading in (1) operates on the temporally spread symbols resulting from (8) rather than on $\mathbf{s}_{l}^{1}, \ldots, \mathbf{s}_{l}^{N_{s}}$. We conclude this section by noting that backward compatibility with IEEE $802.11 \mathrm{a} / \mathrm{g}$ can easily be insured by simply setting the group size to $Q=1$.

\section{ML-based turbo reception}

As in conventional OFDM systems, the reception process on each receive branch (illustrated in Fig. 3) begins by removing the cyclic prefix (CPR) and performing the FFT on each of the OFDM-symbols forming the transmitted frame. In case GO-CDM has been employed at the transmitter, as we assume through the rest 
of this section, the corresponding deinterleaving $\left(\Pi_{2}^{-1}\right)$ is then applied resulting in $N_{r}$ vectors $\mathbf{r}_{l}^{a_{r}}$ of dimension $N_{c}^{d} \times 1$ (pilots and guard subcarriers are not taken into account). These vectors are split into groups, $\mathbf{r}_{g, l}$, in concordance with the grouping performed at transmission. It should be noted now that, as inferred from (7), each received group vector $\mathbf{r}_{g, l}$ contains information from the groups of all transmit spatial streams $\mathrm{s}_{g, l}^{w}$, making mandatory some form of interference cancelling device to reduce the intersymbol interference in order to estimate the different symbol groups from the different spatial streams. The main reason to split the symbols in groups when spreading and multiplexing (i.e. applying group-orthogonality) is to make ML-based detection computationally feasible within each single group. In line with this, we have based our detection mechanism on a bank of list sphere detectors (LSD) [10], each targeting a singlegroup. The LSD detector is based on the sphere detection procedure introduced in [11] which is an efficient method of performing an exhaustive search among a set of candidates (i.e. ML detection). The main feature of LSD is that, apart from symbol estimates, it is able to produce soft information regarding the bits of the estimated symbol in the form of likelihood ratios (LLRs).

Since the LSD detector works on a group basis, it is useful at this point to write the reception equation for a single group as

$$
\left(\begin{array}{c}
\mathbf{r}_{g, l}^{1} \\
\vdots \\
\mathbf{r}_{g, l}^{N_{r}}
\end{array}\right)=\left(\begin{array}{ccc}
\mathbf{H}_{g}^{1,1} \boldsymbol{W} \mathbf{C} & \ldots & \mathbf{H}_{g}^{1, N_{s}} \boldsymbol{W C} \\
\vdots & \ddots & \vdots \\
\mathbf{H}_{g}^{N_{\tau}, 1} \boldsymbol{W} \mathbf{C} & \ldots & \mathbf{H}_{g}^{1, N_{s}} \boldsymbol{W C}
\end{array}\right)\left(\begin{array}{c}
\mathbf{s}_{g, l}^{1} \\
\vdots \\
\mathbf{s}_{g, l}^{N_{s}}
\end{array}\right)+\left(\begin{array}{c}
\boldsymbol{v}_{g, l}^{1} \\
\vdots \\
\boldsymbol{v}_{g, l}^{N_{r}}
\end{array}\right)
$$

or, more compactly,

$$
\tilde{\mathbf{r}}_{g, l}=\mathbf{A}_{g} \tilde{\mathbf{s}}_{g, l}+\tilde{\boldsymbol{v}}_{g, l}
$$

where $\tilde{\mathbf{r}}_{g, l}=\left(\begin{array}{llll}\mathbf{r}_{g, l}^{1} & \ldots & \mathbf{r}_{g, l}^{N_{r}}\end{array}\right)^{T}$ represents the $N_{r}$ reception vectors evaluated on the frequencies assigned to the $g$ th group, $\tilde{\mathbf{s}}_{g, l}=\left(\mathbf{s}_{g, l}^{1} \ldots \mathbf{s}_{g, l}^{N_{s}}\right)^{T}$ are the symbols from all transmitted spatial streams in group $g, \mathbf{A}_{g}$ corresponds to the matrix in (9) jointly modelling the channel, temporal spreading and spatial spreading effects, and $\tilde{\boldsymbol{v}}_{g, l}=\left(\boldsymbol{v}_{g, l}^{1} \ldots \boldsymbol{v}_{g, l}^{N_{r}}\right)^{T}$ are the noise samples added on the group subcarriers. We define now the mapping $\tilde{\mathbf{s}}_{g, l}=\mathcal{M}(\mathbf{b})$ as the modulation mapping to arrive to symbol vector $\tilde{\mathbf{s}}_{g, l}$ comprising all the symbols belonging to group $g$ of the $l$ th OFDM symbol from the $N_{s}$ transmitted spatial streams from the corresponding group bits $\mathbf{b}=\left(\begin{array}{llll}b_{1} & b_{2} & \ldots & b_{\left(N_{s} M Q\right)}\end{array}\right)^{T}$ (to simplify notation, we skip the group and OFDM-symbol indices when referring to the bits). Making use of the Max-log approximation, the LLR for a given bit, $b_{i}$, can then be approximated by $[10]$

$$
\begin{aligned}
\mathcal{L}_{g, l}^{D 1}\left(b_{i}\right) & \approx \frac{1}{2} \max _{\mathbf{b} \in \mathcal{B}_{i,+1}}\left\{-\frac{1}{\sigma_{n}^{2}}\left\|\tilde{\mathbf{r}}_{g, l}-\mathbf{A}_{g} \mathcal{M}(\mathbf{b})\right\|^{2}+\mathbf{b}_{[i]}^{T}\left(\mathcal{L}_{g, l}^{A 1}\right)_{[i]}\right\} \\
& -\frac{1}{2} \max _{\mathbf{b} \in \mathcal{B}_{i,-1}}\left\{-\frac{1}{\sigma_{n}^{2}}\left\|\tilde{\mathbf{r}}_{g, l}-\mathbf{A}_{g} \mathcal{M}(\mathbf{b})\right\|^{2}+\mathbf{b}_{[i]}^{T}\left(\mathcal{L}_{g, l}^{A 1}\right)_{[i]}\right\}
\end{aligned}
$$


where the symbols $\mathcal{B}_{i,+1}$ and $\mathcal{B}_{i,-1}$ represent the sets of $2^{N_{s} M Q-1}$ bit vectors whose $i^{t h}$ position is a ' +1 ' or ' -1 ', respectively. The $\left(N_{s} M Q-1\right) \times 1$ vector $\left(\mathcal{L}_{g, l}^{A 1}\right)_{[i]}$ contains the a-priori LLR for each bit in $\mathbf{b}$ except for the $i^{\text {th }}$ bit. All apriori LLRs are assumed to be zero for the first iteration. Since moderate values of $M$ and/or $Q$ make the sets $\mathcal{B}_{i,+1}$ and $\mathcal{B}_{i,-1}$ extremely large and therefore the search in (11) computationally unfeasible, LSD limits the search to the sets $\hat{\mathcal{B}}_{i,+1}=\mathcal{B}_{i,+1} \cap \mathcal{C}$ and $\hat{\mathcal{B}}_{i,-1}=\mathcal{B}_{i,-1} \cap \mathcal{C}$ where $\mathcal{C}$ is the set containing the bit vectors corresponding to the $N_{\text {cand }}$ group candidates closer, in an Euclidean sense, to the received group vector, i.e., $\mathcal{C}=\left\{\mathbf{b}^{1}, \ldots, \mathbf{b}^{N_{\text {cand }}}\right\}$ where $\mathbf{b}^{j}=$ $\mathcal{M}^{-1}\left(\tilde{\mathbf{s}}_{g, l}^{j}\right)$ with $\left\{\tilde{\mathbf{s}}_{g, l}^{1}, \ldots, \tilde{\mathbf{s}}_{g, l}^{N_{\text {cand }}}\right\}$ being the $N_{\text {cand }}$ group candidates for which

$$
\left\|\tilde{\mathbf{r}}_{g, l}-\mathbf{A}_{g} \tilde{\mathbf{s}}_{g, l}\right\|^{2}
$$

is smallest. Notice that when computing the list of group candidates using (12), the a-priori information from each bit is not taken into account. However, when $N_{\text {cand }}$ is chosen sufficiently large most often the list of candidates minimizing (12) contains with high probability the maximizer (ML solution) of (11). Two points are to be taken into account when applying the LSD. Firstly, it is possible that either $\hat{\mathcal{B}}_{i,+1}$ or $\hat{\mathcal{B}}_{i,-1}$ is empty for a given bit. Such a situation arises when for a given bit position, all the candidates turn out to have a ' +1 ' (or ' 1 ') in that position. In these cases, that bit position is assigned an extreme (positive or negative) value [10]. Secondly, the limitation of the space search to a limited pool of candidates may cause the a-priori information $\left\{\mathcal{L}^{A 1,1}, \ldots, \mathcal{L}^{A 1, N_{s}}\right\}$ to be of limited value or even deleterious whenever the candidate pool does not contain the solution hinted by the a-priori information. This problem can be alleviated using the constrained LSD detector proposed in [12].

The computed LLRs for the bits in the $G$ groups of the $N_{O F D M}$ OFDM-symbols for the $N_{s}$ spatial streams, $\mathcal{L}_{g, l}^{D 1}=\left(\mathcal{L}_{g, l}^{D 1}(1) \ldots \mathcal{L}_{g, l}^{D 1}\left(N_{s} M Q\right)\right)$ are then degrouped and de-segmented to arrange the LLRs in frame format for the $N_{s}$ transmitted streams $\left\{\mathcal{C}^{D 1,1}, \ldots, \mathcal{C}^{D 1, N_{s}}\right\}$. The a-priori information $\mathcal{L}^{A 1}$ is subtracted from the computed LLRs and the resulting extrinsic information $\left\{\mathcal{L}^{E 1,1}, \ldots, \mathcal{L}^{E 1, N_{s}}\right\}$ is then temporally de-interleaved and spatially merged (e.g. inverse spatial parsing) resulting in the signal labeled as $\mathcal{L}^{A 2}$ in Fig. 3, which is fed to a soft-in/soft-out maximum a-posteriori (MAP) decoder. The MAP decoder output can be supplied to a bit slicer to obtain bit estimates or fed back to the LSD module for further LLR refinement. In this latter case, see Fig. 3, only extrinsic information $\left(\mathcal{L}^{E 2}\right)$ is sent back to the detector.

\section{$5 \quad$ Numerical results}

Numerical simulations have been conducted to evaluate the performance of the GO-CDM extension in a IEEE 802.11n setup. The system has been configured to operate on a $20 \mathrm{MHz}$ bandwidth with a total of $N_{c}=64$ subcarriers of which 


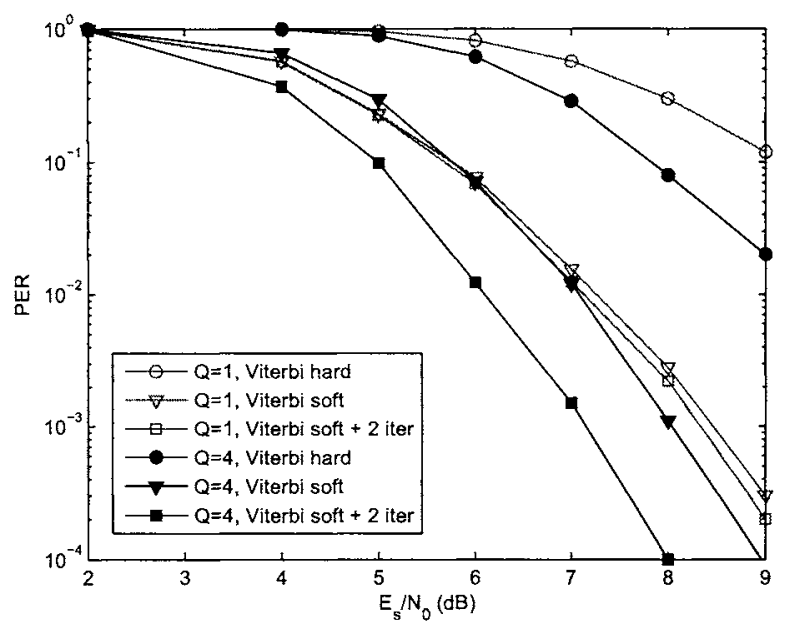

Fig. 4. SDM-CDD transmission. $N_{t}=4, N_{r}=2, N_{s}=2$. MCS 9 .

$N_{c}^{d}=52$ are used to carry data. Without loss of generality, information bits are generated in packets of $N_{b}=416$ bits. The transmitter uses a rate terminated punctured convolutional coder with generator polynomials [133 171]. Perfect channel knowledge and subcarrier synchronisation are assumed at the receiver. It has been also presupposed that the duration of the cyclic prefix appended to each OFDM symbol exceeds the root mean squared channel ( $\mathrm{rms}$ ) delay spread, thus eliminating any interference among consecutive OFDM symbols. As in [7], we have configured the system to have $N_{t}=4$ and $N_{r}=2$ transmit and receive antennae, respectively. The simulations have been conducted using channel model E-NLOS as defined in [13]. This channel profile corresponds to a large office environment and it is made of 38 independent paths distributed among 4 clusters and it has an rms delay spread of $100 \mathrm{~ns}$. Two different spatial configurations have been tested by considering different numbers of spatial streams and particular selections of the spatial spreading matrix. In all simulations, an ML-based turbo reception scheme (with 2 iterations) as the one introduced in the previous section was employed with the number of considered candidates set to $N_{\text {cand }}=64$. For comparison, the results obtained with hard and soft non-iterative Viterbi decoding will also be shown. In the first spatial configuration, the four antennas are configured as two CDD systems (CDD factor $\Lambda=2$ ) operating in parallel. It is easy to see that this setup can be achieved with a choice of spatial spreading matrix given by:

$$
\boldsymbol{W}=\left(\begin{array}{ll}
1 & 0 \\
1 & 0 \\
0 & 1 \\
0 & 1
\end{array}\right)
$$




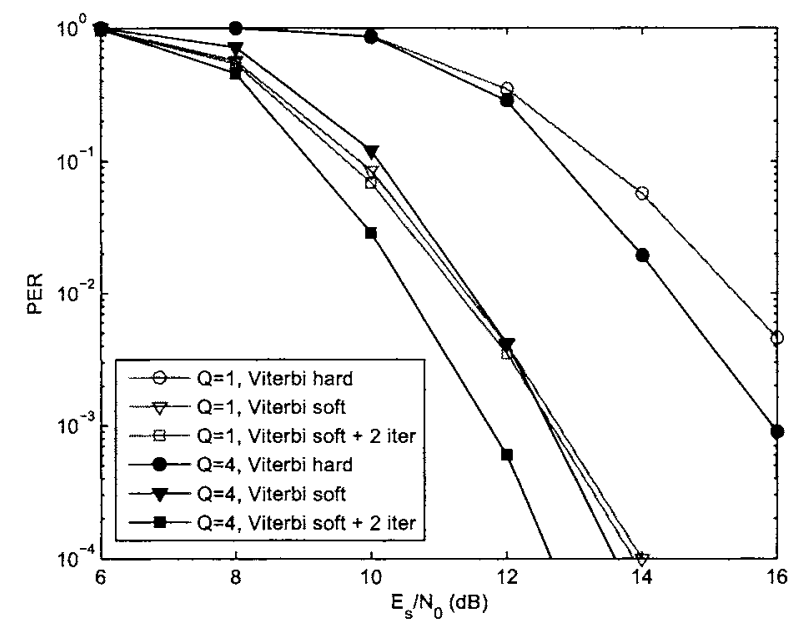

Fig. 5. CDD transmission. $N_{t}=4, N_{r}=2, N_{s}=1$. MCS 3 .

and cyclic delays defined by $\delta_{1}=\delta_{3}=0$ and $\delta_{2}=\delta_{4}=32$. This system is an example of a combination of CDD and SDM, as two independent spatial streams are simultaneously transmitted over different antennae and each stream employs CDD. The system is configured to use QPSK modulation which, in combination with the chosen number of spatial streams and bandwidth, would correspond to the modulation coding scheme (MCS) number 9 in [8] achieving a transmission rate of $26 \mathrm{Mbps}$. Figure 4 shows the attained packet error rate (PER) when employing GO-CDM processing with $Q=4$ (black lines with solid markers) in comparison with the standard system without GO-CDM $(Q=1$, red lines with hollow markers). It can be seen that the configuration with GO-CDM clearly outperforms the conventional system when both employ iterative detection. In particular, gains between 1 and $2 \mathrm{~dB}$ are observed across the range of PER values of practical interest. Note that in the standard configuration, the iterative detection hardly provides any PER reduction over soft Viterbi decoding.

In the second tested configuration only one spatial stream is assumed to be present $\left(N_{s}=1\right)$ and the four transmit antennae are used to perform $\operatorname{CDD}(\Lambda=4)$. Choosing $W=\left(\begin{array}{llll}1 & 1 & 1 & 1\end{array}\right)^{T}$ and setting the cyclic delays to $\delta_{1}=0, \delta_{2}=16$, $\delta_{3}=32$ and $\delta_{4}=48$, configures the system as a 4 -branch CDD setup. For this scenario, the modulation format has been set to 16-QAM (MCS number 3 in [8]) resulting again in a transmission bit rate of $26 \mathrm{Mbps}$. The results with $(Q=4)$ and without $(Q=1)$ GO-CDM are shown in Fig. 5. As in the previous spatial configuration, the simulations outcome leaves no doubt that the system with GOCDM has the potential, using iterative detection, to significantly outperform the current standard. 


\section{Conclusions}

This paper has proposed the introduction of group-orthogonal code-division multiplex within the context of the IEEE 802.11 n (draft) standard. A reception scheme has been developed using an iterative ML-based detection procedure. Simulations results have shown that the combination of GO-CDM with iterative detection brings along important performance gains in terms of $\mathrm{PER}$ reduction irrespective of the spatial configuration used. Moreover, the GO-CDM extension is simple to incorporate to the current standard draft and it can easily be configured to be backward compatible with IEEE $802.11 \mathrm{a} / \mathrm{g}$.

\section{Acknowledgments}

This work has been supported in part by the MEC and FEDER under project MARIMBA (TEC2005-0997), Govern de les Illes Balears under project XISPES and grant PCTIB-2005GC1-09, and a Ramon y Cajal fellowship, Spain.

\section{References}

1. S. Kaiser, "OFDM code-division multiplexing in fading channels," IEEE Trans. Commun., vol. 50, pp. 1266-1273, Aug. 2002.

2. N. Yee, J.-P. Linnartz, and G. Fettweis, "Multi-carrier CDMA in indoor wireless radio networks," in IEEE PIMRC, Yokohama (Japan), Sept. 1993, pp. 109-113.

3. X. Cai, S. Zhou, and G. Giannakis, "Group-orthogonal multicarrier CDMA," IEEE Trans. Commun., vol. 52, no. 1, pp. 90-99, Jan. 2004.

4. F. Riera-Palou, G. Femenias, and J. Ramis, "On the design of group-orthogonal MC-CDMA systems," in Proc. IEEE SPAWC, Cannes (France), July 2006.

5. - , "Downlink performance of group-orthogonal multicarrier systems," in Proc. IFIP PWC, Albacete (Spain), Sept. 2006, pp. 389-400.

6. F. Riera-Palou and G. Femenias, "Combining multicarrier code-division multiplex with cyclic delay diversity for future WLANs," in Proc. IEEE SPAWC, Helsinki (Finland), June 2007, (Accepted for publication).

7. W. Yan, S. Sun, Y. Li, and Y. Liang, "Transmit diversity schemes for MIMOOFDM based wireless LAN systems," in Proc. IEEE PIMRC, Helsinki (Finland), Sept. 2006 , pp. $1-5$.

8. S. A. Mujtaba, "TGn Sync Proposal Technical Specification. doc.:IEEE 802.1104/0889r7," Draft proposal, July 2005.

9. G. Bauch and J. Malik, "Cyclic delay diversity with bit-interleaved coded modulation in orthogonal frequency division multiple access," IEEE Trans. Wireless Commun., vol. 8, pp. 2092-2100, Aug. 2006.

10. B. Hochwald and S. ten Brink, "Achieving near-capacity on a multiple-antenna channel," IEEE Trans. Commun., vol. 51, pp. 389-399, March 2003.

11. U. Fincke and M. Pohst, "Improved methods for calculating vectors of short length in a lattice, including a complexity analysis," Math. Comp., vol. 44, pp. 463-471, Apr. 1985.

12. J. Liu and J. Li, "Turbo processing for an OFDM-based MIMO system," IEEE Trans. Wireless Commun., vol. 4, pp. 1988-1993, Sept. 2005.

13. V. Erceg, "Indoor MIMO WLAN Channel Models. doc.: IEEE 802.11-03/871r0," Draft proposal, Nov. 2003. 